\title{
Conjugate Effect of Radiation and Thermal Conductivity Variation on MHD Free Convection Flow for a Vertical Plate
}

\author{
Rowsanara Akhter ${ }^{1 *}$, Mohammad Mokaddes Ali², Babul Hossain ${ }^{2}$, M. Sharif Uddin ${ }^{1}$ \\ ${ }^{1}$ Department of Mathematics, Jahangirnagar University, Dhaka, Bangladesh \\ ${ }^{2}$ Department of Mathematics, Mawlana Bhashani Science and Technology University, Tangail, Bangladesh \\ Email: *rakhter309@gmail.com, mmali309@gmail.com, babulhossain@yahoo.com, msharifju@yahoo.com
}

Received August 20, 2013; revised September 5, 2013; accepted September 11, 2013

Copyright (C) 2013 Rowsanara Akhter et al. This is an open access article distributed under the Creative Commons Attribution License, which permits unrestricted use, distribution, and reproduction in any medium, provided the original work is properly cited.

\begin{abstract}
A numerical investigation is performed to study the effect of thermal radiation on magnetohydrodynamic (MHD) free convection flow along a vertical flat plate in presence of variable thermal conductivity in this paper. The governing equations of the flow and the boundary conditions are transformed into dimensionless form using appropriate similarity transformations and then solved employing the implicit finite difference method with Keller-box scheme. Results for the details of the velocity profiles, temperature distributions as well as the skin friction, the rate of heat transfer and surface temperature distributions are shown graphically. Results reveal that the thermal radiation is more significant in MHD natural convection flow during thermal conductivity effect is considered. To illustrate the accuracy of the present results, the results for the local skin fraction and surface temperature distribution excluding the extension effects are compared with results of Merkin and Pop designed for the fixed value of Prandtl number and a good agreement were found.
\end{abstract}

Keywords: Radiation; MHD; Thermal Conductivity; Finite Difference Method

\section{Introduction}

The physical phenomenon of free convection flow is driven by temperature difference. Using these considerations, the temperature variation generates a density gradient which responsible for buoyancy forces. The buoyancy effects are important in free convection flow of viscous incompressible electrically conducting fluid. Many practical applications of free convection flow exist, for example in the heater and coolers of mechanical devices, in chemical industries, in nuclear power plants, in the formation of microstructures during the cooling of molten metal's, in fluid flows around heat-dissipation fins, and solar ponds etc. Moreover, MHD free convection flow is used frequently in the field of stellar and planetary magnetospheres, aeronautics, chemical engineering and electronics. Furthermore, most of the engineering processes are related with a high temperature, accordingly, radiation heat transfer is significant to design the relevant equipment of heat transfer process. In addition, radiation effects on MHD free convection flow and heat transfer

\footnotetext{
${ }^{*}$ Corresponding author.
}

are important in the context of space technology. Considering it's important applications in engineering and industrial fields, a number of theoretical and experimental work have been conducted extensively by many researchers. Among them, Soundalgekar and Takhar [1] studied the effect of radiation on MHD free convection flow of a gas past a sami-infinite vertical plate using the Cogley-vincenti-Giles equilibrium model (Cogley et al. [2]). Hossain and Takhar [3] employed implicit finite difference methods to analyze the effect radiation on mixed convection flow along a heated vertical flat plate with a uniform free stream and a uniform surface temperature. The effects of radiation and transverse magnetic field near stretching sheet were investigated by Ghaly [4] in the presence of a uniform free stream of constant velocity, temperature and concentration to show that radiation have significant influences on the velocity and temperature profiles. Abd El-Naby et al. [5] studied the radiation effects on MHD unsteady free convection flow over a vertical plate with variable surface temperature. Badruddin et al. [6] explored the effect of radiation and viscous dissipation on natural convection flow in a porous medium 
by imposing finite element method (FEM). Furthermore, it is also known that the physical property may change significantly with temperature. To obtain better prediction of the flow behavior, it is necessary to take into account this variation of thermal conductivity of fluid. Mishra et al. [7] employed alternating direction implicit scheme and collapsed dimension method to investigate the effect of temperature dependent thermal conductivity and radiation heat transfer on transient conduction for a 2-D rectangular enclosure containing an absorbing, emitting and scattering medium. Seddeek and Salama [8] applied perturbation technique and shooting method to analyze the effects of variable viscosity and thermal conductivity on MHD unsteady two-dimensional laminar flow of a viscous incompressible conducting fluid past a semiinfinite vertical porous moving plate considering variable suction. Sharma and Singh [9] obtained the analytical and numerical solutions of the effects of thermal conductivity on MHD steady free convection flow of a viscous incompressible electrically conducting liquid along an inclined isothermal non-conducting porous plate in presence of viscous dissipation and Ohmic heating. The effects of thermal conductivity on unsteady MHD free convective flow over an isothermal semi infinite vertical plate were studied by Loganathan et al. [10] using implicit finite-difference method of Crank-Nicholson type.

In this paper, the effects of radiation and variable thermal conductivity on free convection flow for a vertical flat plate in presence of transverse magnetic field are studied. The detail derivation of the governing equations for the flow and the parametric discussion depending on the numerical results of the present simulations are presented in the following section.

\section{Governing Equations of the Flow}

We consider the conduction inside a vertical heated flat plate and free convection flow of an incompressible, viscous and electrically conducting fluid along that vertical flat plate of length $l$ and width $b$. The fluid properties are assumed to be constant and the temperature $T_{b}$ of the outer surface of the plate is greater than ambient temperature $T_{\infty}$ and a uniform magnetic field of strength $H_{0}$ is imposed along the $\bar{y}$-axis. Here the $\bar{x}$-axis is taken along the vertical flat plate in upward direction and also the $\bar{y}$-axis is normal to that plate. The effects of radiation from the heated plate and thermal conductivity variation within the two dimensional flow region are considered in this analysis. Moreover, thermal conductivity of the fluid is assumed as $k_{f}=k_{\infty}\left\{1+\gamma^{*}\left(T_{f}-T_{\infty}\right)\right\}$. The flow configuration and the coordinates system are shown in following Figure 1.

The governing equations of the flow under the Boussinesq approximations can be expressed within the usual boundary layer as follows:

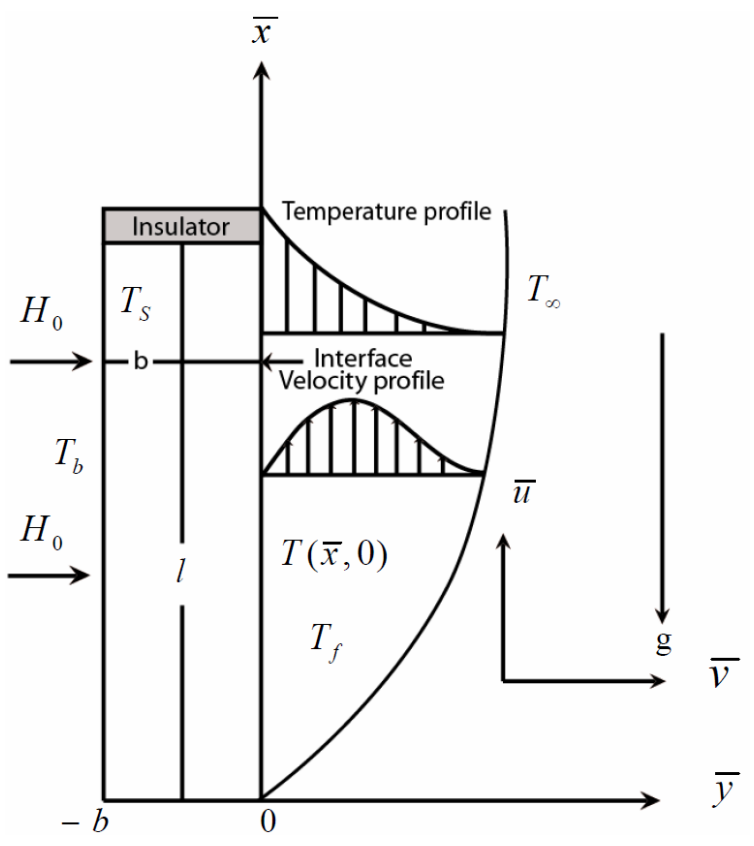

Figure 1. Physical model and coordinate system.

$$
\begin{gathered}
\frac{\partial \bar{u}}{\partial \bar{x}}+\frac{\partial \bar{v}}{\partial \bar{y}}=0 \\
\bar{u} \frac{\partial \bar{u}}{\partial \bar{x}}+\bar{v} \frac{\partial \bar{u}}{\partial \bar{y}}=v \frac{\partial^{2} \bar{u}}{\partial \bar{y}^{2}}+g \beta\left(T_{f}-T_{\infty}\right)-\frac{\sigma H_{0}^{2} \bar{u}}{\rho} \\
\bar{u} \frac{\partial T_{f}}{\partial \bar{x}}+\bar{v} \frac{\partial T_{f}}{\partial \bar{y}}=\frac{1}{\rho C_{p}} \frac{\partial}{\partial \bar{y}}\left(k_{f} \frac{\partial T_{f}}{\partial \bar{y}}\right)-4 \Gamma\left(T_{f}-T_{b}\right)
\end{gathered}
$$

where $\Gamma=\int_{0}^{\infty} K_{\lambda b}\left(\frac{\partial e_{b \lambda}}{\partial T_{f}}\right)_{b} \mathrm{~d} \lambda, K_{\lambda b}=K_{\lambda}\left(T_{b}\right)$ is the mean absorption coefficient [5], $e_{b \lambda}$ is the Plank's function. The boundary conditions based on conduction are:

$$
\left.\begin{array}{l}
\bar{u}=\bar{v}=0, T_{f}=T(\bar{x}, 0), \\
\frac{\partial T_{f}}{\partial \bar{y}}=\frac{k_{s}}{b k_{f}}\left(T_{f}-T_{b}\right) \text { at } \bar{y}=0, \bar{x}>0 \\
\bar{u} \rightarrow 0, T_{f} \rightarrow T_{\infty} \text { at } \bar{y} \rightarrow \infty, \bar{x}>0
\end{array}\right\}
$$

Equations (1) to (3) are nonlinear dimensional partial differential equations and these equations can be made non-dimensional by using the following dimensionless variables:

$$
\left.\begin{array}{l}
x=\frac{\bar{x}}{l}, y=\frac{\bar{y}}{l} G r^{1 / 4}, u=\frac{\bar{u} l}{v} G r^{-1 / 2}, \\
v=\frac{\bar{v} l}{v} G r^{-1 / 4}, \theta=\frac{T_{f}-T_{\infty}}{T_{b}-T_{\infty}}, \\
G r=\frac{g \beta l^{3}\left(T_{b}-T_{\infty}\right)}{v^{2}}
\end{array}\right\}
$$


Therefore, the dimensionless governing equations are:

$$
\begin{gathered}
\frac{\partial u}{\partial x}+\frac{\partial v}{\partial y}=0, \\
u \frac{\partial u}{\partial x}+v \frac{\partial u}{\partial y}+M u=\frac{\partial^{2} u}{\partial y^{2}}+\theta, \\
u \frac{\partial \theta}{\partial x}+v \frac{\partial \theta}{\partial y}=\frac{1}{\operatorname{Pr}}\left\{(1+\gamma \theta) \frac{\partial^{2} \theta}{\partial y^{2}}+\gamma\left(\frac{\partial \theta}{\partial y}\right)\right\} \\
-R a(\theta-1)
\end{gathered}
$$

The corresponding boundary conditions are:

$$
\left.\begin{array}{l}
u=v=0, \theta-1=p \frac{\partial \theta}{\partial y} \text { at } y=0, x>0 \\
u \rightarrow 0, \theta \rightarrow 0 \text { at } y \rightarrow \infty, x>0
\end{array}\right\}
$$

Here $M=\frac{\sigma H_{0}^{2} l^{2}}{\mu} G r^{-1 / 2}$ is the magnetic parameter, $R a=\frac{4 \Gamma l^{2}}{v} G r^{-1 / 2}$ is the radiation parameter,

$\gamma=\gamma^{*}\left(T_{b}-T_{\infty}\right)$ is the thermal conductivity parameter, $\operatorname{Pr}=\frac{\mu C_{p}}{K_{f}}$ is the Prandtl number and $p=\left(k_{f} / k_{s}\right)(b / l) G r^{1 / 4}$ is a conjugate conduction parameter. The value of the conjugate conduction parameter $p$ depends on $(b / l),\left(k_{f} / k_{s}\right)$ and $G r$ but each of which depends on the types of considered fluid and the solid. The steam function and similarity variable and the dimensionless temperature are considered in the following form to solve the governing equations:

$$
\begin{aligned}
& \psi=x^{4 / 5}(1+x)^{-1 / 20} f(x, \eta), \\
& \eta=y x^{-1 / 5}(1+x)^{-1 / 20}, \\
& \theta=x^{1 / 5}(1+x)^{-1 / 5} h(x, \eta)
\end{aligned}
$$

Using the above transformations, we obtain the following dimensionless governing equations:

$$
\begin{gathered}
f^{\prime \prime \prime}+\frac{16+15 x}{20(1+x)} f f^{\prime \prime}-\frac{6+5 x}{10(1+x)} f^{\prime 2} \\
-M x^{2 / 5}(1+x)^{1 / 10} f^{\prime}+h=x\left(f^{\prime} \frac{\partial f^{\prime}}{\partial x}-f^{\prime \prime} \frac{\partial f}{\partial x}\right) \\
\frac{1}{\operatorname{Pr}} h^{\prime \prime}+\frac{\gamma}{\operatorname{Pr}}\left(\frac{x}{1+x}\right) h h^{\prime \prime}+\frac{\gamma}{\operatorname{Pr}}\left(\frac{x}{1+x}\right)\left(h^{\prime}\right)^{2} \\
+\frac{16+15 x}{20(1+x)} f h^{\prime}-\frac{1}{5(1+x)} f^{\prime} h-\operatorname{Rax}^{2 / 5}(1+x)^{1 / 10} h \\
+\operatorname{Rax}^{1 / 5}(1+x)^{3 / 10}=x\left(f^{\prime} \frac{\partial h}{\partial x}-h^{\prime} \frac{\partial f}{\partial x}\right)
\end{gathered}
$$

$$
\begin{aligned}
& \text { The boundary condition (9) become } \\
& \left.\begin{array}{l}
f(x, 0)=f^{\prime}(x, 0)=0 \\
h^{\prime}(x, 0)=-(1+x)^{1 / 4}+x^{1 / 5}(1+x)^{1 / 20} h(x, 0) \text { at } y=0 \\
f^{\prime}(x, \infty) \rightarrow 0, h(x, \infty) \rightarrow 0 \text { at } y \rightarrow \infty \text {. }
\end{array}\right\}
\end{aligned}
$$

In practical point of view, it is important to calculate the values of the skin friction co-efficient in term of surface shear stress and the rate of heat transfer in term of the Nussetl number. These can be written in the dimensionless form as:

$$
C_{f}=\frac{G r^{-3 / 4} l^{2}}{\mu \nu} \tau_{w} \text { and } N u=\frac{G r^{-1 / 4} l}{k_{f}\left(T_{b}-T_{\infty}\right)} q_{w}
$$

where $\tau_{w}=\mu\left(\frac{\partial \bar{u}}{\partial \bar{y}}\right)_{\bar{y}=0}$ is the shearing stress and $q_{w}=-k_{f}\left(\frac{\partial T_{f}}{\partial \bar{y}}\right)_{\bar{y}=0}$ is the heat flux. Thus the local skin friction co-efficient and the local Nussetl number is obtained using the new variable systems that is describes in Equation (20) as follows:

$$
\begin{aligned}
& C_{f x}=x^{2 / 5}(1+x)^{-3 / 20} f^{\prime \prime}(x, 0) \text { and } \\
& N u_{x}=-(1+x)^{-1 / 4} h(x, 0)
\end{aligned}
$$

The numerical value of the surface temperature distribution are obtained from the following relation

$$
\theta(x, 0)=x^{1 / 5}(1+x)^{-1 / 5} h(x, 0)
$$

We have discussed the velocity profiles and temperature distributions for various values of magnetic parameter, radiation parameter, thermal conductivity variation parameter and Prandtl number in the present investigation.

\section{Method of Solution}

The numerical solutions of this analysis are found by using implicit finite difference method with Keller-box [11] Scheme which is well documented by Cebeci and Bradshaw [12].

\section{Comparison of the Results}

The comparison of the skin friction coefficients $\left(C_{f x}\right)$ and the surface temperature $(\theta(x, 0))$ distribution between the present work and the work of Merin and Pop [13] is presented in following Table 1. We observed in this table, that the present analysis is an excellent agreement with the published work.

\section{Results and Discussion}

The main objective of the present work is to analyze 
Table 1. Comparison of the present numerical results of the skin friction $\left(C_{f x}\right)$ and surface temperature $(\theta(x, 0))$ with Prandtl number $\operatorname{Pr}=1.00$, and $p=1.00$.

\begin{tabular}{ccccc}
\hline \multirow{2}{*}{$x^{\frac{1}{5}}=\xi$} & \multicolumn{2}{c}{ Merin and Pop [13] } & \multicolumn{2}{c}{ Present work (2013) } \\
\cline { 2 - 5 } & $C_{f x}$ & $\theta(x, 0)$ & $C_{f x}$ & $\theta(x, 0)$ \\
\hline 0.7 & 0.430 & 0.651 & 0.424 & 0.651 \\
0.8 & 0.530 & 0.686 & 0.529 & 0.687 \\
0.9 & 0.635 & 0.715 & 0.635 & 0.716 \\
1.0 & 0.745 & 0.741 & 0.744 & 0.741 \\
1.1 & 0.859 & 0.762 & 0.860 & 0.763 \\
1.2 & 0.972 & 0.781 & 0.975 & 0.781 \\
\hline
\end{tabular}

MHD free convection flow in presence of thermal conductivity variation with radiation effects. In this analysis, the numerical solutions are calculated from the transformed momentum and energy equations. The value of conjugate conduction parameter $p=1.0$ is considered for the simulation of the present problem and the values of Prandtl number are considered 0.733, 0.930, 1.241 and 1.630 which corresponds to air, ammonia water and glycerin, respectively. The detailed numerical solutions have been obtained in terms of velocity, temperature, local skin friction, heat transfer rate and surface temperature for a wide range of values of the parameters as $M=0.10,0.50$, 0.70 and 1.00, $R a=0.01,0.03,0.06$ and 0.08, $\gamma=0.01$, $0.10,0.15$ and 0.20 and then presented graphically in Figures 2-11, respectively.

The numerical values of velocity and temperature are obtained from the solution of the Equations (11) and (12) with the boundary condition (13) for different values of magnetic parameter $M$ when $P r=0.733, R a=0.01$ and $\gamma$ $=0.01$ and are illustrated in Figures 2(a) and (b), respectively. Here we observed that the velocity decreases for the increasing values of $M$. This is to be expected because, the magnetic field acting along the horizontal direction that introduces a retard force due to the interaction between applied magnetic field and fluid flow which acts against the fluid motion, as a result, the velocity of the fluid decreases. But near the surface of the plate the velocity increases and become maximum and then decrease and finally approaches to zero. Moreover, the velocity profiles meet together after certain position of $\eta$ and cross the side. This is because, the gradient of decreasing of velocity decrease with the increasing value of magnetic parameter. In Figure 2(b), the temperature within the boundary layer increases with the increasing values of magnetic parameter $M$ due to the interaction of applied magnetic field and fluid motion that tends to heat the fluid. Furthermore, the temperature decreases monotonically with increasing of $\eta$ for each value of $M$. Thus the magnetic field works to retard the fluid motion but increase the temperature within the thermal boundary layer region.

The variation of velocity and temperature for distinct values of the radiation parameter $R a$ together with an
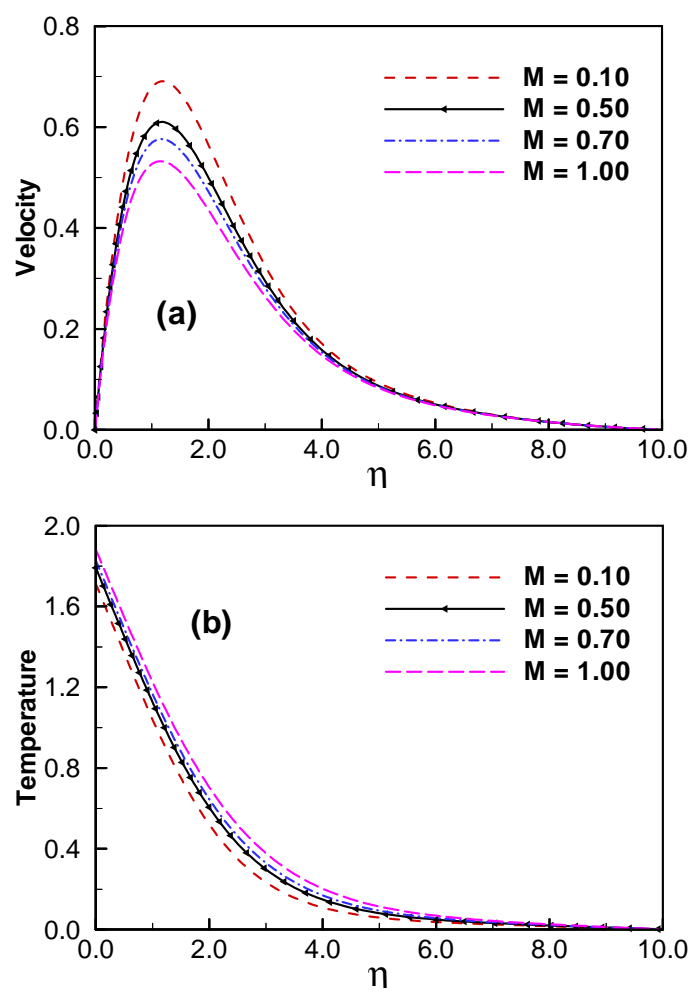

Figure 2. (a) Variation of velocity and (b) Variation of temperature against $\eta$ for varying of $M$ with $R a=0.01 \gamma=0.01$, and $\operatorname{Pr}=0.733$.

individual certain value of Prandtl number, $P r$, magnetic parameter, $M$ and thermal conductivity variation parameter, $\gamma$ are presented in Figures 3(a) and (b), respectively. It can be seen that both the velocity and the temperature of the fluid increase within the velocity boundary layer and the temperature boundary layer, respectively for the increasing $R a$ due to the absorbsion of emitted heat from the heated plate that caused by the radiation effect. The trend is observed to shift upward and the peak velocity increases gradually with the increasing values of $R a$. As the velocity and temperature of the fluid increases with the increasing value of radiation parameter as shown in Figures 3(b) and (b), the thickness of the velocity and thermal boundary layer increase.

Figures 4(a) and (b) illustrate the effects of thermal conductivity variation parameter on velocity and temperature profiles associated with the certain value of $M$, $R a$ and $P r$. Both figures reflect that the velocity and the temperature of the fluid increases with the increasing value of $\gamma$. The fact behind it's that the increasing value of thermal conductivity increases the energy transfer ability. It is also seen that near the surface of the plate the velocity becomes maximum with increasing of $\gamma$ then after the peak position start to decrease and finally approaches to zero. On the other hand, the maximum values of temperature are occurred on the surface of the plate for each 

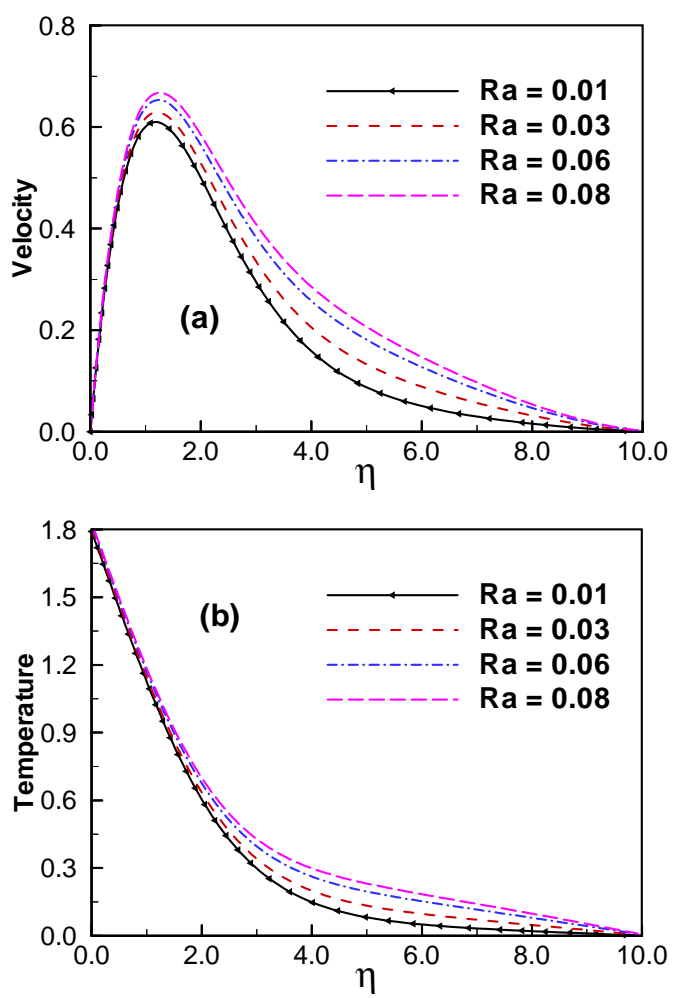

Figure 3. (a) Variation of velocity and (b) Variation of temperature against $\eta$ for varying of $R a$ with $M=0.50 \gamma=0.01$, and $\operatorname{Pr}=\mathbf{0 . 7 3 3}$.
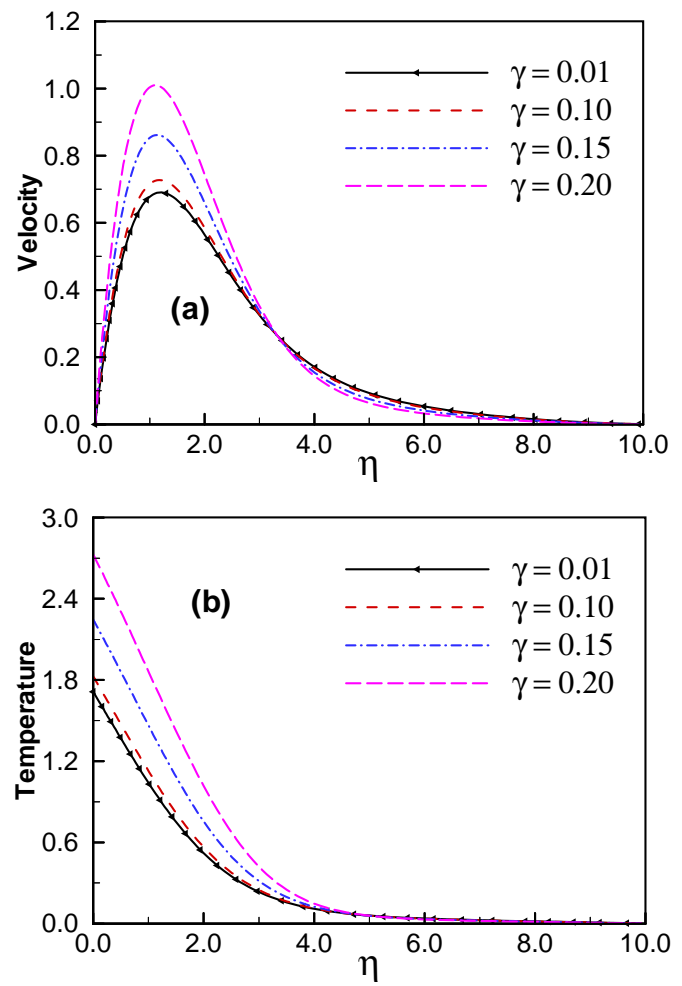

Figure 4. (a) Variation of velocity and (b) Variation of temperature against $\eta$ for varying of $\gamma$ with $M=0.50, R a=0.01$ and $\operatorname{Pr}=\mathbf{0 . 7 3 3}$. value of $\gamma$ and then turn to decrease asymptotically and finally approach to zero. These phenomenons are demonstrated in Figures 4(a) and (b), respectively.

The effect of $\operatorname{Pr}$ on the velocity and temperature distributions is displayed in Figures 5(a) and (b), respectively. From Figure 5(a) observed that the velocity of the fluid decrease as $P r$ increases. It is due to the fact that for increasing $P r$, density of the fluid increases which creates a negative force to flow and then fluid does not move freely. Furthermore, Figure 5(b) shows that the temperature profiles for change in $\mathrm{Pr}$ from 0.733 to 1.630 and seen that the thermal boundary layer thickness decrease for increasing $P r$, because of the increased $P r$ decrease the thermal diffusivity, which leads to the decrease of the energy transfer ability.

Figures 6(a) and (b), respectively reveal that the skin friction coefficient and the heat transfer rate for some selected values of $M$ with $P r=0.733, R a=0.01$, and $\gamma=0.01$. The increased value of $M$ leads to decrease the skin friction along the plate due to the fact the effect of magnetic field parameter opposes the fluid flow. Increasing fluid temperature for increasing $M$ decrease the rate of heat transfer from the plate to fluid. This is because, the increased temperature reduces the temperature difference between the heated plate and fluid within the boundary layer.
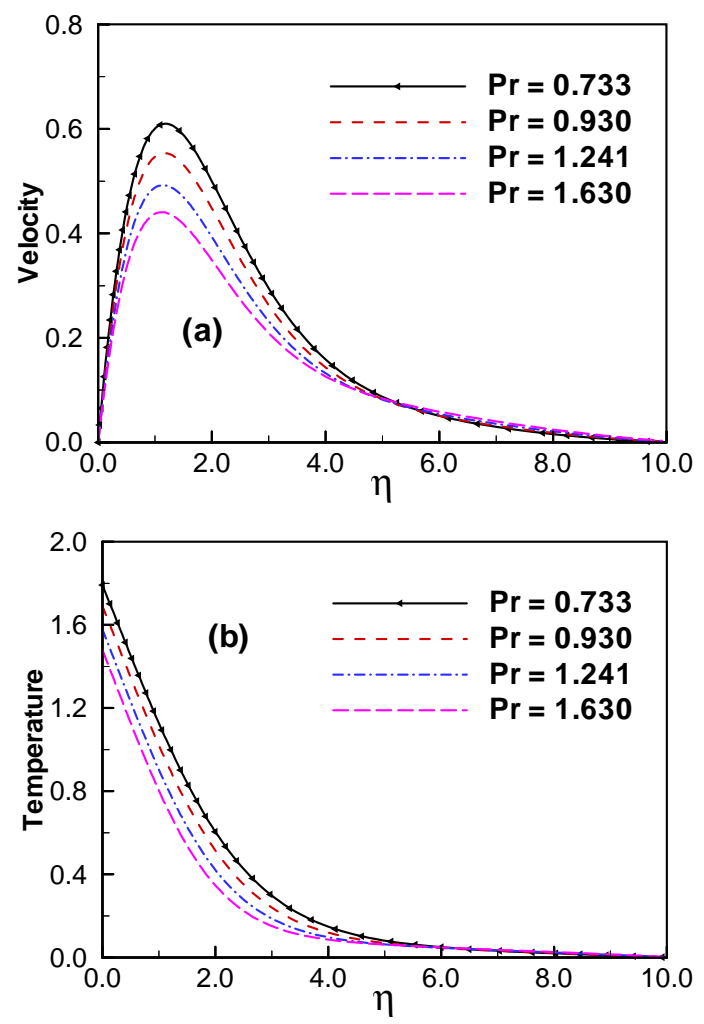

Figure 5. (a) Variation of velocity and (b) Variation of temperature against $\eta$ for varying of $\operatorname{Pr}$ with, $M=0.50, R a=$ 0.01 and $\gamma=0.01$. 

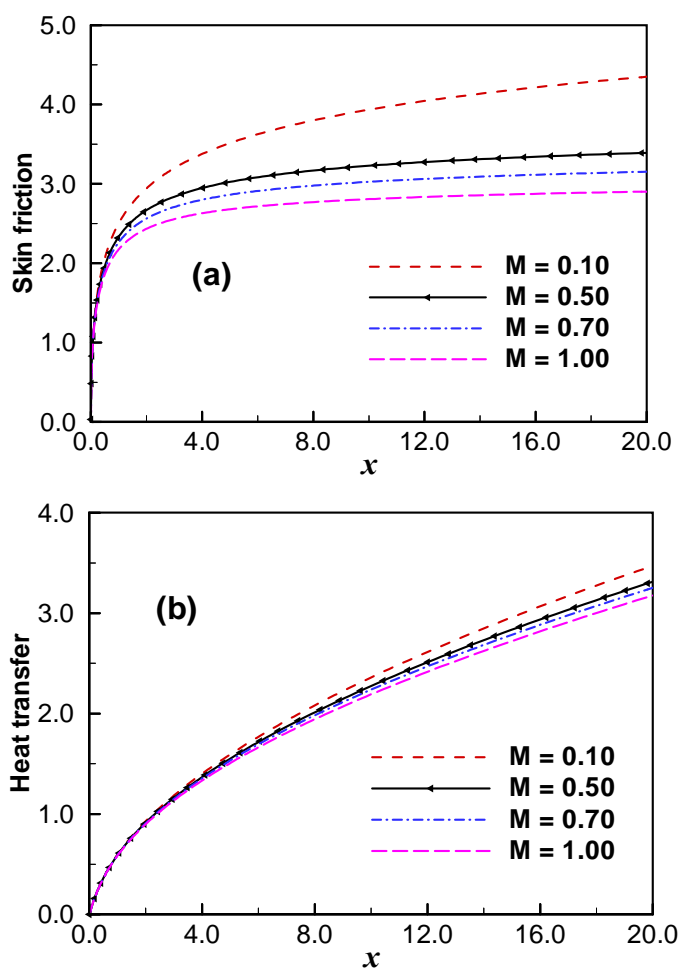

Figure 6. (a) Variation of skin friction and (b) Variation of heat transfer against $x$ for varying of $M$ with $R a=0.01, \gamma=$ 0.01 and $\operatorname{Pr}=0.733$.

Figures 7(a) and (b) plotted the numerical values of the local skin friction coefficient $\left(C_{f x}\right)$ and heat transfer rate $\left(N u_{x}\right)$ for different values of $R a$ associated with the distinct values of controlling parameter. As the radiation effect increases the fluid motion as well as temperature within the boundary layer which are mentioned earlier in Figures 3(a) and (b), respectively. Accordingly, the corresponding skin friction increases and heat transfer rate decreases with the increasing value of $R a$ along the $x$ direction.

The variation of skin friction and heat transfer rate for the effect of conductivity variation parameter are depicted in Figures 8(a) and (b), respectively. The increasing value of $\gamma$ generates greater buoyancy force which therefore increases the friction between the inner surface of the vertical plate and moving fluid particles. Thus the skin friction increases for the greater value of $\gamma$ that is demonstrate in Figure 8(a). Moreover, an increase in the value of $\gamma$ leads to increase the energy transfer ability within the flow region, as a result, the heat transfers rate increases with the increasing of $\gamma$.

The effects of Prandtl number on the skin friction $\left(C_{f x}\right)$ and heat transfer rate $\left(N u_{x}\right)$ against $x$ for the fixed values of $M, R a$ and $\gamma$ are shown respectively in Figures 9(a) and (b). The increased values of $P r$ decrease both the velocity and temperature of the fluid within the boundary layer, consequently, the related skin friction on the plate decreases but the heat transfer rate from heated plate to fluid increases that has been exposed in Figures 9(a) and
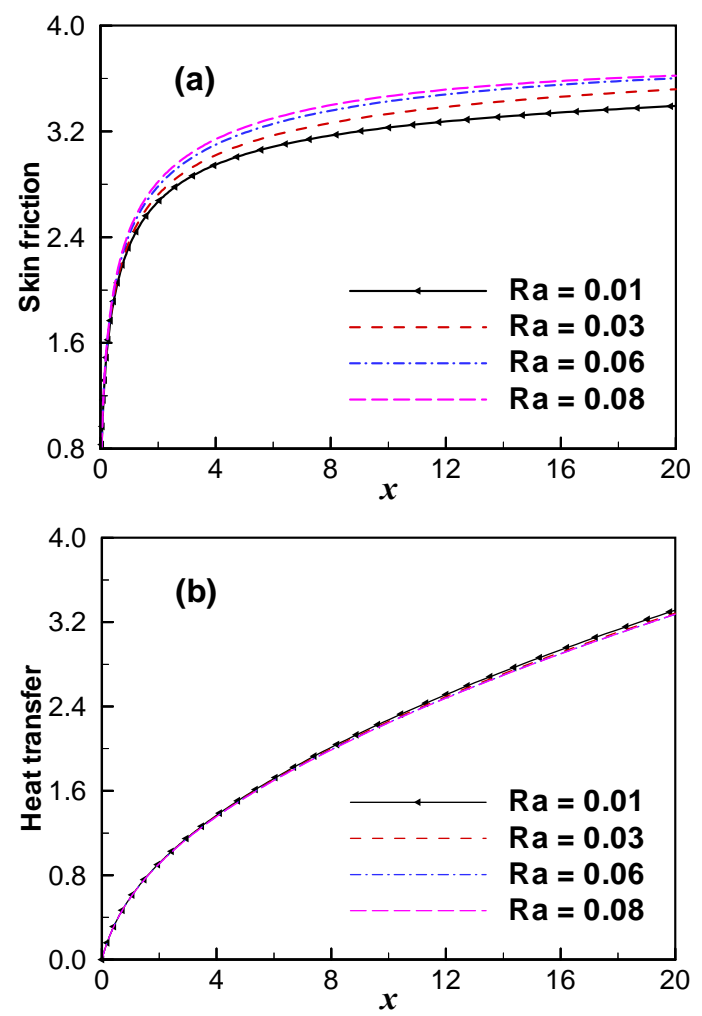

Figure 7. (a) Variation of skin friction and (b) Variation of heat transfer against $x$ for varying of $R a$ with $M=0.50, \gamma=$ 0.01 and $P r=0.733$.
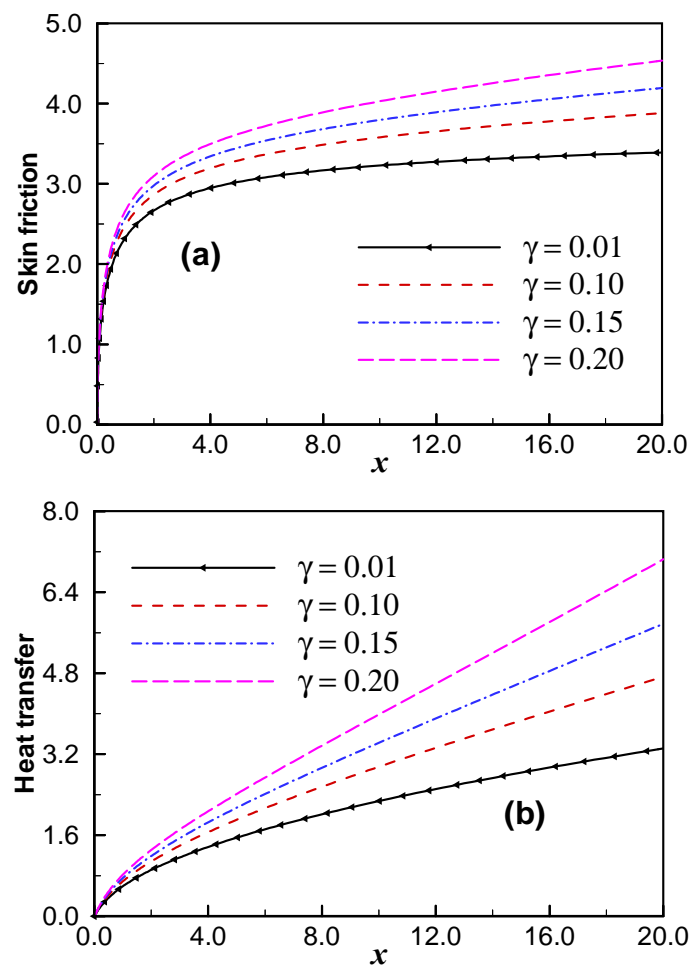

Figure 8. (a) Variation of skin friction and (b) Variation of heat transfer against $x$ for varying of $\gamma$ with $M=0.50, R a=$ 0.01 and $P r=0.733$. 

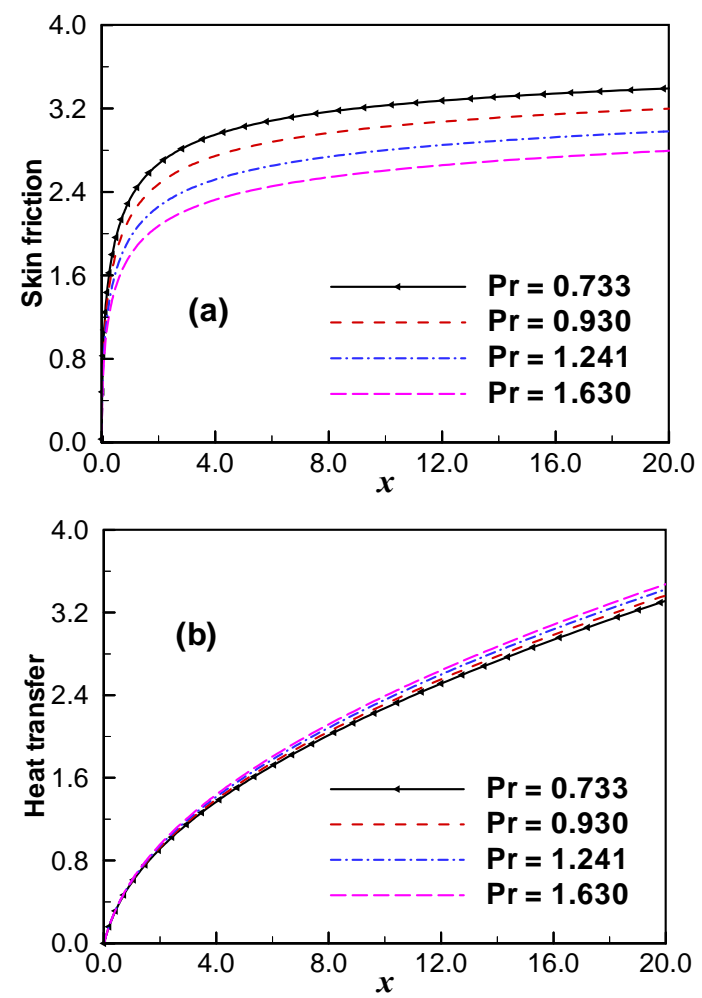

Figure 9. (a) Variation of skin friction and (b) Variation of heat transfer against $x$ for varying of $\operatorname{Pr}$ with $M=0.50, R a=$ 0.01 and $\gamma=0.01$.

(b). Moreover, for a particular value of $P r$, the local skin friction coefficient and heat transfer rate increase monotonically along the $x$ direction.

The influence of magnetic field parameter, radiation parameter, conductivity variation parameter and Prandtl number on the interfacial temperature are depicted in Figures 10 and 11, respectively. The overall temperature profiles shift upward as well as the thermal boundary layer thickness increases with the increasing values of $M$, $R a$, and $\gamma$ observed in Figures 2(b), 3(b) and 4(b), respectively. Consequently, the surface temperature increase for enlarging values of $M, R a$ and $\gamma$, respectively. On the other hand, increasing $\operatorname{Pr}$ decreases the fluid temperature which results interfacial temperature decreases that is shown in Figure 11(b).

\section{Conclusions}

In the present analysis, we have studied numerically the effects of radiation on MHD free convection flow under the influence of thermal conductivity variation for a vertical flat plate and the numerical solutions of the transformed governing equations associated with the specified boundary are obtained for different values of related physical parameters including magnetic parameter, radiation parameter, thermal conductivity variation parameter and Prandtl number. The particular conclusions in this
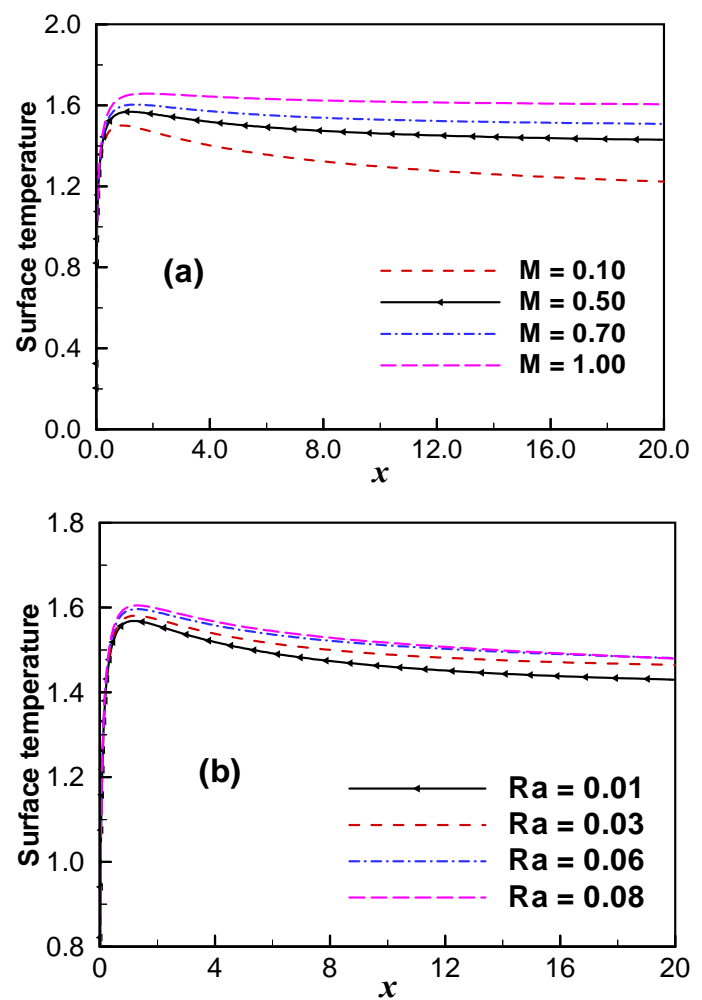

Figure 10. (a) Variation of surface temperature against $x$ for varying $M$ and (b) Variation of surface temperature against $\boldsymbol{x}$ for varying of $\boldsymbol{R a}$.
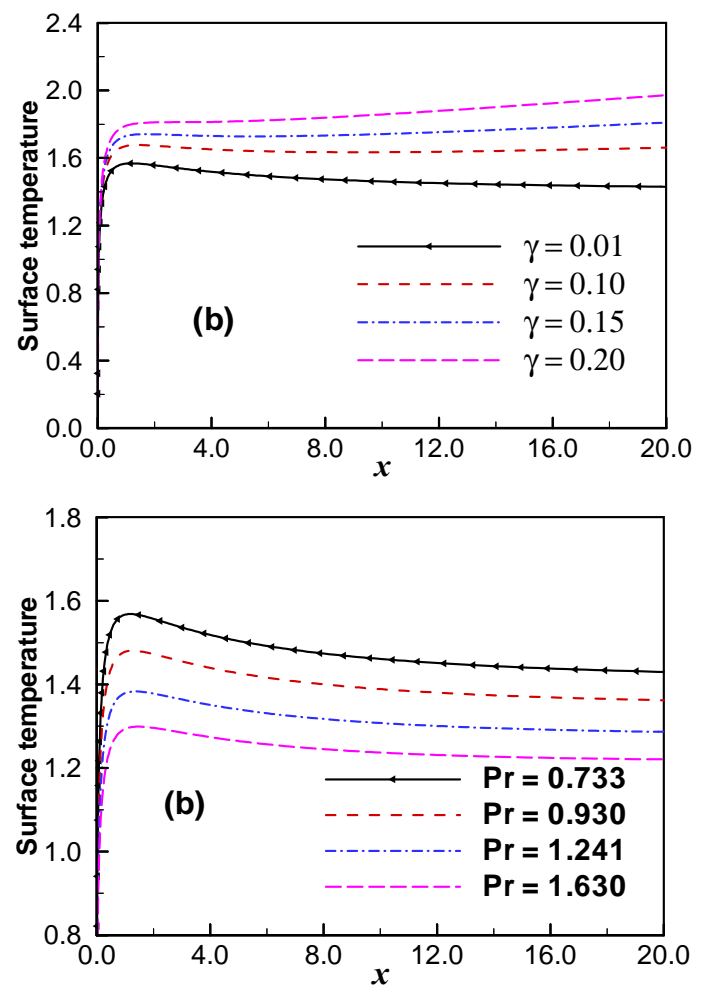

Figure 11. (a) Variation of surface temperature against $x$ for varying $\gamma$ and (b) Variation of surface temperature against $x$ for varying of $\boldsymbol{P r}$. 
study can be listed as follows:

The velocity of the fluid within the boundary layer and the skin friction at the interface increase for decreasing values of magnetic parameter, $M$, Prandtl number, $P r$ and increasing values of the radiation parameter, $R a$ and thermal conductivity variation parameter, $\gamma$.

The increasing value of $M, R a$ and $\gamma$ leads to increase in the value of temperature within the thermal boundary layer as well as the surface temperature on the plate and the effect of $M$ and $R a$ decrease heat transfer rate from plate to fluid within the boundary layer but opposite results hold for increasing of $\gamma$ and $P r$.

\section{REFERENCES}

[1] V. M. Soundalgekar and H. S. Takhar, "Radiative Convective Flow Past a Semi-Infinite Vertical Plate,” Modelling Measurement and Control, Vol. 51, 1992, pp. 31-40.

[2] A. C. Cogley, W. G. Vincenti and S. E. Giles, "Differential Approximation for Radiative in a Non-Gray Gas Near Equilibrium," American Institute of Aeronautics and Astronautics Journal, Vol. 6, No. 3, 1968, pp. 551-553. http://dx.doi.org/10.2514/3.4538

[3] M. A. Hossain and H. S. Takhar, "Radiation Effect on Mixed Convection along a Vertical Plate with Uniform Surface Temperature," Heat and Mass Transfer, Vol. 31, No. 4, 1996, pp. 243-248. http://dx.doi.org/10.1007/BF02328616

[4] A. Y. Ghaly, "Radiation Effects on a Certain MHD Free Convection Flow," Chaos, Solitons \& Fractals, Vol. 13, No. 9, 2002, pp. 1843-1850. http://dx.doi.org/10.1016/S0960-0779(01)00193-X

[5] M. A. Abd El-Naby, E. M. E. Elsayed and N. Y. Abdelazem, "Finite Difference Solution of Radiation Effect on MHD Unsteady Free Convection Flow over a Vertical Plate Variable Surface Temperature,” Journal of Applied Mathematics, Vol. 2003, No. 2, 2003, pp. 65-86. http://dx.doi.org/10.1155/S1110757X0320509X

[6] I. A. Badruddin, Z. A. Zainal, A. Zahid Khan and Z. Mallick, "Effect of Viscous Dissipation and Radiation on Na- tural Convection in a Porous Medium Embedded within Vertical Annulus," International Journal of Thermal Science, Vol. 46, No. 3, 2007, pp. 221-227. http://dx.doi.org/10.1016/j.ijthermalsci.2006.05.005

[7] S. C. Mishra, P. Talukdar, D. Trimis and F. Durst, “TwoDimensional Transient Conduction and Radiation Heat Transfer with Temperature Dependent Thermal Conductivity," International Communications in Heat and Mass Transfer, Vol. 32, No. 3-4, 2005, pp. 305-314. http://dx.doi.org/10.1016/j.icheatmasstransfer.2004.05.015

[8] M. A. Seddeek and F. A. Salama, "The Effects of Temperatute Dependent Viscosity and Thermal Conductivity on Unsteady MHD Convective Heat Transfer Past a SemiInfinite Vertical Porous Moving Plate with Variable Suction," Computational Material Science, Vol. 40, No. 2, 2007, pp. 186-192.

http://dx.doi.org/10.1016/j.commatsci.2006.11.012

[9] P. R. Sharma and G. Singh, "Effects of Variable Thermal Conductivity, Viscous Dissipation on Steady MHD Natural Convection Flow of Low Prandtl Fluid on an Inclined Porous Plate with Ohmic Heating,” Meccanica, Vol. 45, No. 2, 2010, pp. 237-247. http://dx.doi.org/10.1007/s11012-009-9240-0

[10] P. Loganathan, P. Ganesan and D. Iranian, "Effects of Thermal Conductivity on Unsteady MHD Free Convective Flow over a Semi Infinite Vertical Plate,” International Journal of Engineering Science and Technology, Vol. 2, 2010, pp. 6257-6268.

[11] H. B. Keller, "Numerical Methods in the Boundary Layer Theory," Annual Review of Fluid Mechanics, Vol. 10, 1978, pp. 417-433. http://dx.doi.org/10.1146/annurev.fl.10.010178.002221

[12] T. Cebeci and P. Bradshow, "Physical and Computational Aspects of Convective Heat Transfer," Springer, New York, 1984. http://dx.doi.org/10.1007/978-3-662-02411-9

[13] J. H. Merkin and I. Pop, "Conjugate Free Convection on a Vertical Surface,” International Journal of Heat and Mass Transfer, Vol. 39, No. 7, 1996, pp. 1527-1534. http://dx.doi.org/10.1016/0017-9310(95)00238-3

\section{Nomenclature}

$C_{f x}$ : Local skin friction coefficient

$C_{p}$ : Specific heat at constant pressure

$f$ : Dimensionless stream function

$g$ : Acceleration due to gravity

$G r$ : Grashof number

$h$ : Dimensionless temperature

$k_{f}, k_{s}$ : Fluid and solid thermal conductivities

$M$ : Magnetic parameter

$N u_{x}$ : Local Nusselt number

$p$ : Conjugate conduction parameter

Pr: Prandtl number
$T_{f}$ : Temperature of the fluid

$\bar{u}, \bar{v}:$ Velocity components

$u, v$ : Dimensionless velocity components

$\bar{x}, \bar{y}$ : Cartesian co-ordinates

$x, y$ : Dimensionless Cartesian co-ordinate

$\beta$ : Coefficient of thermal expansion

$\gamma$ : Thermal conductivity variation parameter

$\eta$ : Dimensionless similarity variable

$\theta$ : Dimensionless temperature

$\mu, v$ : Dynamic and kinematic viscosities

$\rho$ : Density of the fluid

$\sigma$ : Electrical conductivity 